\title{
Taxing sin goods and subsidizing health care*
}

Helmuth Cremer, Toulouse School of Economics (IDEI and Institut universitaire de France).

Philippe De Donder, Toulouse School of Economics (GREMAQ-CNRS and IDEI).

Dario Maldonado, Department of Economics and CeiBA-Complejidad, Universidad del Rosario, Bogota.

Pierre Pestieau, CREPP, HEC-Management School University of Liège; CORE, Université Catholique de Louvain.

Abstract: We study the taxation of sin goods in a two-period, three-good model. Individuals can buy health care to compensate for the damages caused by their earlier sin good consumption. Individuals are myopic and underestimate the effect of the sinful consumption on health; in their second period, they may acknowledge their mistake or persist in their error. We characterize and compare the first-best and the (linear) second-best taxes in these different settings. In particular, we examine how the results are affected by the way sin good consumption and health care interact in the health production technology.

Keywords: paternalism, behavioral economics, dual self vs single self

JEL classification: H21, I18

* We thank participants to Queen's University's 2007 Public Economics Workshop for helpful suggestions. We are also grateful to the referees for their comments and remarks. Part of this paper was written while the second author was visiting Yale University. He thanks Yale Economics Department for its hospitality. This paper has been financed by the french Agence Nationale de la Recherche's POLNUTRITION grant (ANR-05-PNRA-012, Politiques Nutritionnelles, Régulation des Filières Alimentaires et Consommation). We thank ANR for its support. 


\section{Introduction}

In our everyday life we consume a number of goods that all bring us utility. For most of them, that is all. For some, today's consumption can also have some effects on tomorrow's health. For example, smoking leads to shorter lives or excess sugar to diabetes. To the extent that we impose costs on ourselves, there is no need for government action except if, out of ignorance or myopia, we do not take into account the delayed damage done to our health. ${ }^{1}$ If this is the case, then there is a "paternalistic" mandate for public action, assuming that the government has a correct perception of the health damage generated by our sinful consumption.

Optimal sin taxes have been studied by O’Donoghue and Rabin (2003, 2006). They model an economy where individuals have hyperbolic preferences and differ in both their taste for the sin good and in their degree of time-inconsistency. They show how (heterogeneity in) time inconsistency affects the optimal (Ramsey) consumption tax policy. Their main insight is that, "although taxes create consumption distortion for fully selfcontrolled people, such distortions are second-order relative to the benefits from reducing over-consumption by people with self-control problems" (O'Donoghue and Rabin, 2006, p. 1827). Gruber and Koszegi (2001) study a Pigouvian tax used to counteract overconsumption due to self-control problems, and apply their model to the determination of optimal cigarette taxes. Gruber and Koszegi (2004) also study cigarette taxation with self-control problems, but their focus is the tax incidence for different income groups rather than optimal taxes.

O'Donoghue and Rabin $(2003,2006)$ are representative of the literature studying present-biased preferences (such as Laibson (1997)) in two respects. First, they assume

1 We are not concerned here by two important issues: addiction and externalities associated with sinful activities. 
that all biased individuals disapprove or regret their past consumption decisions and, second, there is nothing that agents can do to mitigate the current impact of past consumption decisions. Our paper lifts these two assumptions and studies their consequences on optimal sin taxes.

We model a two-period setting where individuals consume a sin good, with positive immediate gratification but negative impact on second period health status. In the second stage, individuals may invest in health care services that have a positive impact on their health status. Individuals differ in income and in their awareness of the link between sin good consumption and health care on the one hand, and health status on the other hand.

We contrast two possibilities. In the first one, individuals in their second period realize the mistake they committed previously. They regret their past high sin good consumption, and invest in health care understanding its correct impact on their health and utility. In other words, individuals suffer from myopia in the first period, but use their true or correct preferences later on when they choose health expenditures. They thus exhibit "dual selves", using a term coined in the behavioral economics literature. A second case, referred to as "persistent error", occurs when all decisions, including the determination of health expenditures, are made according to the mistaken preferences. In other words, when individuals realize their error (in the middle of the second period) it is too late to correct for it.

We use the concept of sinful consumption in a narrower sense than other authors. We are not interested by externalities such as those related to passive smoking. We are only concerned by the damage the sinful consumption exerts on the consumer's own health. Sinful consumption is part of the lifestyles regarded as risk factors, the so-called "Holy Four", namely tobacco smoking, drinking of alcoholic beverage, eating an unhealthy diet 
(fat, salt, sweets) and lack of physical activity. ${ }^{2}$

In our setting, sin goods have the following features: they have detrimental effects on health, their consumers do not fully anticipate these effects, they bring regret with some lag and they can be partially offset by costly treatment. The issue of regret is documented by a number of recent surveys. For example, Fong et al. (2004) show on the basis of telephone surveys in Canada, the US, the UK and Australia that about $90 \%$ of smokers agree with the statement: "If you had to do it again, you would not have started smoking". Finally there is the issue of ex post compensatory treatment. In some instances there exists no treatment, or there is no choice as in the case of emphysema that requires oxygen therapy. In many other instances, however, there are treatments that can partially alleviate the problems brought about by one of the Holy Four.

Note that the formal structure of our model can be applied to account for circumstances that go beyond sinful consumption. It can, for example, apply to situations where habit leads to unforeseen consequences. For instance, people can face needs for which they are not prepared and which can force them to work longer than expected or even to unretire. $^{3}$

Which preferences should the social planner use when assessing optimal taxes/subsidies on sin good consumption and health care expenditures? The recent literature on paternalism has studied the impact of behavioral considerations on the social objective. Thaler and Sunstein (2003) make a strong case for "libertarian paternalism", which applies when no coercion is involved, such as when the planner chooses the default option (for example, automatic enrollment in 401(k) employee savings plans in the US). Other papers 2 See Harris (1999). There may be other risk factors but there is less research into their prevalence or into their importance for the burden of disease.

3 See Cremer et al. (2008). 
go further and envision the possibility of coercing individuals by taxing certain goods or even prohibiting their consumption. The literature has focused upon the case where people differ in their degree of non-rationality. These contributions advocate the use by the planner of "cautious" (O’Donoghue and Rabin (1999)) or "asymmetric" (Camerer et al. (2003)) paternalism, which trades-off the benefits of paternalistic interventions for people making mistakes against the costs for fully rational individuals. This literature shows that this kind of paternalism usually leads to some intervention, because deviations from laissez-faire impose second-order costs on rational individuals, but imply first-order gains for non-rational persons. Moreover, it is shown (O'Donoghue and Rabin (2003, 2006)) that even a small probability (or proportion) of people making mistakes can have dramatic effects for optimal policy.

We depart from this literature in two ways. First, in addition to studying the dual self setting, we consider a paternalistic objective where individuals are adamant in their mistakes - i.e., where they either never realize (for instance because of ignorance or cognitive dissonance) or realize too late that they base their decisions on wrong premises. ${ }^{4}$ Second, rather than mixing rational and non-rational individuals, we contrast the results obtained when all individuals are repentant in the second period or when none is. Obviously, paternalism is easier to defend when individuals have dual selves. Similarly, it does not appear to be problematic in the case of "persistent" errors if individuals eventually realize they made a mistake (albeit too late to take any corrective measures). When mistakes are truly persistent and individuals never realize their mistakes we return to an

4 Aronsson and Thunström (2008) and Aronsson and Sjögren (2009) also analyze settings where individuals consume a sinful good and can invest in health capital. Their analysis differs from ours in several ways. Both papers introduce myopia or ignorance in a different way than we do and which does not allow for the introduction of the distinction of persistent errors and dual self. Additionally, the first paper does not consider the issue of the complementarity/subsitutability of sin good consumption and health expenditure on health stock and they only consider first best settings. The second paper introduces non-linear taxation instead of linear taxation. 
analysis similar to the older literature on "merit goods" (Musgrave (1959), more recently Besley (1988)), where we add that the reason for the difference between the planner's and the individuals' preferences resides in the (unrecognized) mistakes made by individuals.

To keep the analysis tractable, we make a number of simplifying assumptions. First, we assume that individuals live two periods. Consequently, we cannot have hyperbolic preferences which require a three period specification. However, their essential feature, namely the regrets for yielding to the short term concerns, is represented in our setting. ${ }^{5}$ Second, we study linear tax instruments. Non-linear instruments are clearly more general but at the same time they constrain the analysis to focus on a limited number of individuals. ${ }^{6}$

We obtain the following main results. We show that the first-best outcome can be decentralized with individualized linear taxes and subsidies in the two scenarios (persistent error and dual self). In the first one, it is necessary to tax the sin good consumption while subsidizing health care expenditures. There is no need to influence saving. The second scenario is more complex, because the social planner faces a problem with changing preferences. The planner has to intervene in the first period by taxing the sin good while subsidizing savings. There is no need to influence health care expenditures, which are optimally chosen provided that first period choices are optimal. Comparing the sin tax in the two scenarios, we obtain that it is smaller in the dual self case if and only if the marginal effect of health care on health status increases with sin good consumption. We also show that under this same condition the possibility of compensating health expenditure makes the sin tax smaller in the dual-self case setting.

5 Additionally in our setting the distinction between sophisticated and non sophisticated individuals is irrelevant as we do not have any commitment device. For example, in political economy models, sophisticated individuals can constrain their short term self by their vote (see Cremer et al., 2007 and Haavio and Kotakorpi, 2009).

6 See Cremer et al. (2009) and Blomquist and Micheletto (2006). 
We then turn to the second best setting where the planner observes neither income, preferences nor savings and uses uniform lump sum transfer, and taxes/subsidies on the sin good and on health expenditure. In the single self case, optimal linear sin taxes and health expenditure subsidies depend upon two terms: a (classical) covariance term reflecting distributive considerations and a "Pigouvian" term that reflects the "internalities" an individual imposes on himself. In the dual self setting the optimal tax formulas also contain a third term, which is linked to the inability to control savings. This additional term would call for higher tax on sin good/subsidy on health care provided that this tax/subsidy encourages savings.

The rest of the paper is organized as follows. In section II, the model, the firstbest solution and the decentralization conditions are presented for the two specifications. Then in section III we turn to the second-best problem when individuals persist in their ignorance. In section IV, we study the alternative second-best problem, that is when individuals realize having made a mistake. A final section concludes.

\section{First-best and decentralization}

\section{Model}

We consider a society consisting of $I$ types of individuals indexed by subscript $i$. Each type of individual is characterized by a wealth endowment $w_{i}$ and subjective and objective health parameters $\alpha_{i}$ and $\beta_{i}$. The proportion of type $i$ individuals in the population is given by $n_{i}\left(\sum_{i=1}^{I} n_{i}=1\right)$. Each individual's life spans two periods. In the first one, he consumes a numeraire good $c_{i}$ and a sin good $x_{i}$. He also saves $s_{i}$ for future expenses. In the second period, he consumes an amount $d_{i}$ of the numeraire and he invests $e_{i}$ in health improvement. In this second period, he enjoys a quality of health $\beta_{i} h\left(x_{i}, e_{i}\right)$, 
on which $x_{i}$ has a negative effect and $e_{i}$ a positive effect. For reasons of ignorance or myopia, the individual has a perception of this function that underestimates the impact of both arguments. In other words, he perceives a health function equal to $\alpha_{i} h\left(x_{i}, e_{i}\right)$ with $\alpha_{i}<\beta_{i}$. We assume for the moment that individuals underestimate the function $h($.$) in both periods (persistent error).$

His two-period utility function can be written as:

$$
U_{i}=u\left(c_{i}\right)+\varphi\left(x_{i}\right)+u\left(d_{i}\right)+\alpha_{i} h\left(x_{i}, e_{i}\right),
$$

with budget constraints:

$$
\begin{aligned}
w_{i} & =(1+\tau) s_{i}+(1+\theta) x_{i}+c_{i}-a_{i}, \\
d_{i} & =s_{i}-(1+\sigma) e_{i},
\end{aligned}
$$

where $u$ and $\varphi$ are strictly concave functions, $\tau, \theta, \sigma$ are tax rates and $a_{i}$ is a lump sum transfer. For simplicity, we assume a zero time discount rate and a zero rate of interest.

Here the myopia parameter concerns the health function. An alternative specification could be $h\left(\alpha_{i} x_{i}, e_{i}\right)$, in which case myopia only concerns the sin good and not health care. This would not change the qualitative nature of our results. A third approach, often used in papers on social security and saving, is to assume that myopia concerns the whole second period, namely the utility of health but also the utility for second period consumption. In that case, the utility function would be given by

$$
u\left(c_{i}\right)+\varphi\left(x_{i}\right)+\alpha_{i}\left[u\left(d_{i}\right)+h\left(x_{i}, e_{i}\right)\right]
$$

\section{First-best}

We assume that the government is paternalistic utilitarian. In other words, its objective consists of the sum of utilities (1) in which $\beta_{i}$ replaces $\alpha_{i}$. As a benchmark, we derive 
the first-best (FB) conditions by maximizing the following Lagrangian expression

$$
\mathcal{L}_{1}=\sum_{i=1}^{I} n_{i}\left[u\left(c_{i}\right)+\varphi\left(x_{i}\right)+u\left(d_{i}\right)+\beta_{i} h\left(x_{i}, e_{i}\right)-\mu\left(c_{i}+x_{i}+d_{i}+e_{i}-w_{i}\right)\right],
$$

where $\mu$ is the multiplier associated with the resource constraints. The FOCs yield:

$$
u^{\prime}\left(c_{i}\right)=u^{\prime}\left(d_{i}\right)=\varphi^{\prime}\left(x_{i}\right)+\beta_{i} h_{x}\left(x_{i}, e_{i}\right)=\beta_{i} h_{e}\left(x_{i}, e_{i}\right)=\mu
$$

with $h_{x}<0$ and $h_{e}>0$. Denote the first-best solution by $c_{i}^{*}, x_{i}^{*}, d_{i}^{*}$ and $e_{i}^{*}$. We can also define $s_{i}^{*}=d_{i}^{*}+e_{i}^{*}$, the (implicit) individual savings at the first-best solution.

The utilitarian planner equalizes marginal utility of consuming the numeraire good in both periods. Since preferences for this good are the same for all individuals, this calls for $c_{i}^{*}$ and $d_{i}^{*}$ to be equal and the same for all. Marginal utility for numeraire and sin goods are also equalized, with the latter composed of the immediate marginal gratification and of the (true) delayed marginal impact on health. Finally, the planner also equalizes second period marginal utility from consuming the numeraire good and from consuming health care. If the marginal impact of sin good consumption on health is the same for all individuals $\left(\beta_{i}=\beta\right)$, then $x_{i}^{*}$ and $e_{i}^{*}$ are also identical for all.

We now contrast the first-best solution with the laissez-faire allocation obtained when individuals maximize their own utility. We show that the first-best allocation can be decentralized with individualized (redistributive) lump sum taxes and Pigouvian taxes or subsidies. We have to distinguish two settings: persisting errors on the one hand, and dual self on the other hand. 


\section{Decentralization with persisting errors}

With persisting errors there is just one optimization problem at the start of the first period. It amounts to maximizing:

$$
\begin{aligned}
U_{i}= & u\left(w_{i}-(1+\tau) s_{i}-(1+\theta) x_{i}+a_{i}\right) \\
& +\varphi\left(x_{i}\right)+\alpha_{i} h\left(x_{i}, e_{i}\right)+u\left(s_{i}-(1+\sigma) e_{i}\right) .
\end{aligned}
$$

which yields the following FOCs

$$
\begin{aligned}
& -(1+\tau) u^{\prime}\left(c_{i}\right)-u^{\prime}\left(d_{i}\right)=0 \\
& -(1+\theta) u^{\prime}\left(c_{i}\right)+\varphi^{\prime}\left(x_{i}\right)+\alpha_{i} h_{x}\left(x_{i}, e_{i}\right)=0 \\
& -(1+\sigma) u\left(d_{i}\right)+\alpha_{i} h_{e}\left(x_{i}, e_{i}\right)=0 .
\end{aligned}
$$

In the laissez-faire (LF), $\tau=\sigma=\theta=a_{i}=0$ and we have

$$
u^{\prime}\left(c_{i}\right)=u^{\prime}\left(d_{i}\right)=\varphi^{\prime}\left(x_{i}\right)+\alpha_{i} h_{x}\left(x_{i}, e_{i}\right)=\alpha_{i} h_{e}\left(x_{i}, e_{i}\right) .
$$

Marginal utility of consuming the numeraire and the sin goods are also equalized (though they differ across agents if there is heterogeneity in $\alpha_{i}$ ), but not at the correct level since individuals make a mistake when assessing the impact of both sin good and health care consumption on their second period utility (health status).

To decentralize the first-best optimum, we need individualized redistributive lump sum taxes $a_{i}$ and individualized corrective taxes or subsidies on the sin good and health expenditure. Combining (2) with (3)-(5) yields the following lemma.

Lemma 1 In the case of persistent errors, it is possible to decentralize the first-best allocation by using individualized lump sum taxes together with a tax on sin good and a 
subsidy on compensatory health given by

$$
\begin{aligned}
\theta_{i} & =\frac{\left(\alpha_{i}-\beta_{i}\right) h_{x}\left(x_{i}^{*}, e_{i}^{*}\right)}{u^{\prime}\left(c_{i}\right)}>0 \\
\sigma_{i} & =\frac{\left(\alpha_{i}-\beta_{i}\right)}{\beta_{i}}<0 .
\end{aligned}
$$

No tax or subsidy on savings is needed and we have $\tau=0$.

The tax on sin good consumption forces the individual to internalize the full impact of his sin good consumption on his health. It is proportional to the share (given by the difference between $\beta_{i}$ and $\alpha_{i}$ ) of the marginal impact of sin good on health that he does not spontaneously internalize. It is also necessary to subsidize health care, since individuals underestimate its impact on health. Intuitively, the subsidy rate is equal to the percentage of underestimation by the individual $\left(\beta_{i}-\alpha_{i}\right) / \beta_{i}$. There is no need to influence saving, since individuals do not exhibit time-inconsistent preferences. The simplicity of formula (7) is due to the specification adopted: additive utilities and multiplicative myopia parameter.

The taxes, $\theta_{i}$, and subsidies, $\sigma_{i}$, are individualized as long as the parameter $\alpha$ or $\beta$ varies across individuals. Naturally, with $\alpha_{i}=\alpha$ and $\beta_{i}=\beta$, taxes would be identical for all.

\section{Decentralization with dual self}

In the previous subsection we have assumed that individuals stick to their beliefs in the second period when they choose $e$. Let us now make the reasonable assumption that in the second period they realize that they have made a mistake out of ignorance or myopia and take their decision concerning health care using their correct preferences. In behavioral economics, one then speaks of dual self. 
When the "reasonable" self prevails in the second period, the choice of $e_{i}$ is determined by the equality

$$
(1+\sigma) u^{\prime}\left(s_{i}-(1+\sigma) e_{i}\right)=\beta_{i} h_{e}\left(x_{i}, e_{i}\right)
$$

However, this level of $e$ is not the one that the individual envisioned when he chose his sin good consumption and saving in the first period. The amount of health care that the individual originally planned to buy, denoted by $e_{i}^{P}$, is given by

$$
(1+\sigma) u^{\prime}\left(s_{i}-(1+\sigma) e_{i}^{P}\right)=\alpha_{i} h_{e}\left(x_{i}, e_{i}^{P}\right)
$$

The levels of $s_{i}$ and $x_{i}$ then satisfy the following first-order conditions:

$$
\begin{aligned}
& -\left(1+\tau_{i}\right) u^{\prime}\left(c_{i}\right)+u^{\prime}\left(s_{i}-e_{i}^{P}\right)=0, \\
& -\left(1+\theta_{i}\right) u^{\prime}\left(c_{i}\right)+\varphi^{\prime}\left(x_{i}\right)+\alpha_{i} h_{x}\left(x_{i}, e_{i}^{P}\right)=0 .
\end{aligned}
$$

Is it possible to decentralize the first-best optimum in these conditions with our linear instruments that are chosen in the first period? Combining (2) with equations (8)-(11) shows that this is possible using $\tau_{i}$ and $\theta_{i}$ plus $a_{i}$. With these instruments, and denoting optimal values with $\mathrm{a}^{*}$, one obtains $x_{i}^{*}$ and $s_{i}^{*}$, which then imply $e_{i}^{*}$. Defining $e_{i}^{P *}$ as the planned level of $e_{i}$ when the tax instruments are set to decentralize the first-best, we obtain the levels of the tax and subsidy that decentralize the first-best in this case. These levels are shown in the following lemma.

Lemma 2 In the case of dual self, it is possible to decentralize the first-best allocation by using individualized lump sum taxes together with a subsidy on savings and a tax on the sin good given by

$$
\begin{aligned}
\tau_{i} & =\frac{u^{\prime}\left(s_{i}^{*}-e_{i}^{P *}\right)-u^{\prime}\left(c_{i}^{*}\right)}{u^{\prime}\left(c_{i}^{*}\right)} \\
\theta_{i} & =\frac{\alpha_{i} h_{x}\left(x_{i}^{*}, e_{i}^{P *}\right)-\beta_{i} h_{x}\left(x_{i}^{*}, e_{i}^{*}\right)}{u^{\prime}\left(c_{i}^{*}\right)}
\end{aligned}
$$


No tax or subsidy on health expenditures is needed and we have $\sigma=0$.

Equation (8) shows that the individual will take the optimal health care decision in the second stage, provided that he chose the optimal values of $x_{i}$ and $s_{i}$ in the first stage. Influencing the health care decision is then unnecessary, provided that tax instruments on saving and sin good consumption decentralize these two optimal choices. The sin tax is proportional to the mistake made by the individual. This mistake comes from two sources: under-estimation of the impact of $\sin$ good on health (since $\alpha_{i}<\beta_{i}$ ) and misplanning of the future amount of health care consumed $\left(e_{i}^{P}\right.$ as opposed to $\left.e_{i}\right)$. Since individuals misplan their future health care need, it is also necessary to influence their saving decision, as shown by (12). ${ }^{7}$

\section{Comparison of sin taxes in the two specifications}

It is interesting to compare the sin taxes obtained under the two specifications. To make the comparison easier, we assume that $\alpha_{i}=\alpha>0$ and $\beta_{i}=\beta>\alpha$. Note that, under this assumption, the use of a personalized lump sum transfer will make all individuals demand the same amounts of all goods $\left(c_{i}^{*}=c^{*}, x_{i}^{*}=x^{*}, e_{i}^{*}=e^{*}, e_{i}^{P *}=e^{P *}\right)$. We thus have (with $S$ for single self and $D$ for dual self):

$$
\begin{aligned}
\theta^{S} & =\frac{(\alpha-\beta) h_{x}\left(x^{*}, e^{*}\right)}{u^{\prime}\left(c^{*}\right)} \\
\theta^{D} & =\frac{(\alpha-\beta) h_{x}\left(x^{*}, e^{*}\right)+\alpha\left[h_{x}\left(x^{*}, e^{P *}\right)-h_{x}\left(x^{*}, e^{*}\right)\right]}{u^{\prime}\left(c^{*}\right)} .
\end{aligned}
$$

In the two cases, the sin tax is proportional to the error made in the first stage when

7 Assuming $\alpha_{i}=\alpha$ and $\beta_{i}=\beta$, we obtain $\tau_{i}=\tau$ and $\theta_{i}=\theta$. In words, $a_{i}$ makes everyone identical and the Pigouvian tax and subsidy rates are identical. To illustrate this point, assume a single individual with $\alpha=0<\beta=1$. We then have $e_{i}^{P *}=0$ so that the implementing tax rates or subsidy are

$$
\tau=\frac{u^{\prime}\left(s^{*}\right)}{u^{\prime}\left(c^{*}\right)}-1<0 \quad \text { and } \quad \theta=\frac{-h_{x}\left(x^{*}, e^{*}\right)}{u^{\prime}\left(c^{*}\right)}>0 .
$$


evaluating the damage of sin good consumption on health, measured at the optimal sin good and health care consumptions. An additional term is present in the dual self case, which is proportional to the second mistake made by the individual in that case. Since this individual misestimates how much health care he will buy at the optimum, he is also mistaken in his assessment of the marginal damage done by the optimal amount of sin good consumption, as measured by the function $h(x, e)$. The sign of this impact depends on the cross-derivative of this function. Assume for instance that it is positive. Since the individual under-estimates how much health care he will buy, he then over-estimates how bad the marginal impact of sin good will be ${ }^{8}$ (as measured by the function $h($.$) ). This calls$ for decreasing the tax on the sin good, compared to a "single self" individual. Assuming that $h_{x e}$ has everywhere the same sign, we then obtain the following proposition

Proposition 1 In the first-best, if $\alpha_{i}=\alpha>0$ then

$$
\theta^{S} \lesseqgtr \theta^{D} \quad \Longleftrightarrow \quad h_{x e} \lesseqgtr 0
$$

Proposition 1 says that the sin tax is higher (lower) in the case of dual self than in the case of persistent error if the marginal productivity of health expenditures decreases (increases) with the consumption of the sin good. Consequently the comparison depends on the sign of the cross derivative. A positive sign means that the marginal effectiveness (productivity) of health care expenditures increases with the consumption of the sin good; with a negative sign, health care expenditures are less effective for higher sin good consumption levels. The sign of this cross derivative depends upon the kind of sin good under consideration. For instance, it seems reasonable to assume that it is positive if the sin good is sugar: the more you eat, the more medications designed to treat diabetes may be helpful to you. With this assumption the sin tax is smaller when the individual 8 Recall that $h_{x}()<$.0 . 
acknowledges his mistake in the second period of his life. Given the increased productivity of health care, in the dual case the individual easily corrects for his earlier excess while the individual in the persistent errors case is unable or unwilling to do so. The opposite assumption can be made for smoking: heavy smokers increase their probability of getting lung cancer, for which there is up to now no efficient cure in the majority of cases. Put bluntly, there is not much utility that you can get from consuming health care if you end up with lung cancer following heavy smoking.

So far we have contrasted the first-best policies with dual self on the one hand and persistent error on the other hand. However, it is also interesting to study the impact of the availability of a compensatory treatment. To do this, we compare the policy with dual self when a compensatory treatment exists and when it does not; let $\theta_{n c}^{D}$ represent the optimal sin tax when there is no compensatory treatment in the dual self case. To make this comparison we have to set the value of $e$ to get a meaningful counterfactual. The most natural approach is to adopt the optimal value of $e$, that is $e^{*}$ such as defined in the first-best section, and to assume that it is exogenous. In that case, it is plain that the optimal sin tax is exactly the same as the one with persistent error, specified by equation (6), i.e.

$$
\theta_{n c}^{D}=\frac{(\alpha-\beta) h_{x}\left(x^{*}, e^{*}\right)}{u^{\prime}\left(c^{*}\right)}
$$

This tax rate, by its very definition, induces the individual to choose the optimal level of the sin good for given health expenditures, $e^{*}$. Recall that in the case of persistent error, the planned and effective levels of $e$ are equal and no correction for the misplanning of $e$ is needed in the rule for the sin tax. When there is no compensatory treatment, such a correction is of course not needed either. In other words, in the case of persistent ignorance there is no willingness to correct for the sin good induced health deterioration; 
when there is no compensatory treatment there is no room for correction. This leads to the following proposition.

Proposition 2 In the case of dual self, if $\alpha_{i}=\alpha>0$ then

$$
\theta_{n c}^{D} \lesseqgtr \theta^{D} \quad \Longleftrightarrow \quad h_{x e} \lesseqgtr 0
$$

Proposition 2 says that the possibility of compensating for the sinful consumption leads to a higher (lower) first-best sin tax (compared to a setting where such a compensation is not available) if the marginal productivity of health expenditures decreases (increases) with the consumption of the sin good.

\section{Second-best in the case of persistent errors}

We now turn to the second-best setting with linear tax instruments and a uniform lump sum transfer. It is the combination of these two assumptions which explains the move to a second best setting. We assume in the remaining of this paper that $\beta_{i}=\beta>\alpha_{i}$. In other words, the objective effect of both $e$ and $x$ on health is the same for all, but individuals differ in their degree of myopia (as well as in income). We also assume that taxes/subsidies on saving are not available anymore (either because saving is not observable, or because elements not modeled, like international mobility of capital, prevent saving from being taxed or subsidized). ${ }^{9}$ This allows us to concentrate on the two instruments which are the most relevant from the perspective of this paper.

We first consider the case where the individuals never acknowledge that the true health parameter is $\beta$ (or do so after $e$ is determined). In that case, restricting the instruments

9 Introducing this extra instrument would complicate the expressions considerably without affecting the results in a significant way. In particular, even with the three tax instruments (but without non uniform lump-sum transfers) the first best cannot be implemented except in very special cases; see below for additional discussion. 
to linear taxes and uniform lump sum transfer we write the new Lagrangian as

$$
\begin{aligned}
\mathcal{L}_{2}= & \sum_{i=1}^{I} n_{i}\left[u\left(w_{i}-s_{i}-x_{i}(1+\theta)+a\right)+\varphi\left(x_{i}\right)+u\left(s_{i}-(1+\sigma) e_{i}\right)\right. \\
& \left.+\beta h\left(x_{i}, e_{i}\right)-\mu\left(a-\theta x_{i}-\sigma e_{i}\right)\right],
\end{aligned}
$$

where $s_{i}, x_{i}$ and $e_{i}$ are functions of $a, \theta$ and $\sigma$ and are obtained from the following optimal conditions for individual choices:

$$
\begin{aligned}
& -u^{\prime}\left(c_{i}\right)+u^{\prime}\left(d_{i}\right)=0, \\
& -u^{\prime}\left(c_{i}\right)(1+\theta)+\varphi^{\prime}\left(x_{i}\right)+\alpha_{i} h_{x}\left(x_{i}, e_{i}\right)=0, \\
& -u^{\prime}\left(d_{i}\right)(1+\sigma)+\alpha_{i} h_{e}\left(x_{i}, e_{i}\right)=0 .
\end{aligned}
$$

The first-order conditions for the maximization of $\mathcal{L}_{2}$ are provided in Appendix A. In compensated terms, these expressions can be written as: ${ }^{10}$

$$
\begin{aligned}
& \frac{\partial \tilde{\mathcal{L}}_{2}}{\partial \theta}=-\operatorname{cov}\left(u^{\prime}\left(c_{i}\right), x_{i}\right)+\sum_{i=1}^{I} n_{i}\left(\beta-\alpha_{i}\right) H_{\theta i}+\mu \sum_{i=1}^{I} n_{i}\left[\theta \frac{\partial \tilde{x}_{i}}{\partial \theta}+\sigma \frac{\partial \tilde{e}_{i}}{\partial \theta}\right]=0, \\
& \frac{\partial \tilde{\mathcal{L}}_{2}}{\partial \sigma}=-\operatorname{cov}\left(u^{\prime}\left(c_{i}\right), e_{i}\right)+\sum_{i=1}^{I} n_{i}\left(\beta-\alpha_{i}\right) H_{\sigma i}-\mu \sum_{i=1}^{I} n_{i}\left[\theta \frac{\partial \tilde{x}_{i}}{\partial \sigma}+\sigma \frac{\partial \tilde{e}_{i}}{\partial \sigma}\right]=0,
\end{aligned}
$$

where

$$
H_{\theta i}=h_{x}\left(x_{i}, e_{i}\right) \frac{\partial \tilde{x}_{i}}{\partial \theta}+h_{e}\left(x_{i}, e_{i}\right) \frac{\partial \tilde{e}_{i}}{\partial \theta} \quad \text { and } \quad H_{\sigma i}=h_{x}\left(x_{i}, e_{i}\right) \frac{\partial \tilde{x}_{i}}{\partial \sigma}+h_{e}\left(x_{i}, e_{i}\right) \frac{\partial \tilde{e}_{i}}{\partial \sigma}
$$

It is important to note that we here use the concept of average compensation and not that of the standard Slutsky term. Using a tilde $(\sim)$ for our compensation term and a 10 Defining

$$
\frac{\partial \tilde{\mathcal{L}}_{2}}{\partial \theta}=\frac{\partial \mathcal{L}_{2}}{\partial \theta}+\frac{\partial \mathcal{L}_{2}}{\partial a} \bar{x}_{i} \quad \text { and } \quad \frac{\partial \tilde{\mathcal{L}}_{2}}{\partial \sigma}=\frac{\partial \mathcal{L}_{2}}{\partial \sigma}+\frac{\partial \mathcal{L}_{2}}{\partial a} \bar{e}_{i}
$$

where $\bar{x}_{i}$ and $\bar{e}_{i}$ are the average values of $x_{i}$ and $e_{i}$, respectively. Additionally, recall that for any two variables $z_{i}$ and $v_{i}$ defined for our population

$$
\operatorname{cov}\left(z_{i}, v_{i}\right)=\sum_{i=1}^{I} n_{i} z_{i} v_{i}-\bar{z}_{i} \bar{v}_{i}
$$


hat $\left({ }^{\wedge}\right)$ for the standard Slutsky term, we have

$$
\begin{aligned}
\frac{\partial \tilde{x}_{i}}{\partial \sigma} & =\frac{\partial x_{i}}{\partial \sigma}+\frac{\partial x_{i}}{\partial a} \bar{e}_{i}=\frac{\partial \hat{x}_{i}}{\partial \sigma}+\frac{\partial x_{i}}{\partial a}\left(\bar{e}_{i}-e_{i}\right), \\
\frac{\partial \tilde{x}_{i}}{\partial \theta} & =\frac{\partial \hat{x}_{i}}{\partial \theta}+\frac{\partial x_{i}}{\partial a}\left(\bar{x}_{i}-x_{i}\right) .
\end{aligned}
$$

Our approach is simple, but the signs of the compensated terms have to be interpreted with caution. For example, we know that $\partial \hat{x}_{i} / \partial \theta<0$, but if $x_{i}$ is much smaller than the average, $\bar{x}_{i}, \partial \tilde{x}_{i} / \partial \theta$ could be positive.

In interpreting the above FOCs, we assume that these own compensated derivatives are negative. ${ }^{11}$ Observe that with identical individuals the first-best optimum is obtained with just $\sigma$ and $\theta$. Equations (19) and (20) jointly define the values of $\theta$ and $\sigma$. To obtain the tax formulas that define each of these taxes/subsidies we can use Cramer's rule to solve for $\theta$ and $\sigma$ to obtain

$$
\begin{aligned}
& \theta=\frac{1}{D_{p e}}\left[\operatorname{cov}\left(u^{\prime}\left(c_{i}\right), x_{i}\right) \sum_{i=1}^{I} n_{i} \frac{\partial \tilde{e}_{i}}{\partial \sigma}-\operatorname{cov}\left(u^{\prime}\left(c_{i}\right), e_{i}\right) \sum_{i=1}^{I} n_{i} \frac{\partial \tilde{e}_{i}}{\partial \theta}\right. \\
& \left.-\sum_{i=1}^{I} n_{i}\left(\beta-\alpha_{i}\right) H_{\theta i} \sum_{i=1}^{I} n_{i} \frac{\partial \tilde{e}_{i}}{\partial \sigma}+\sum_{i=1}^{I} n_{i}\left(\beta-\alpha_{i}\right) H_{\sigma i} \sum_{i=1}^{I} n_{i} \frac{\partial \tilde{e}_{i}}{\partial \theta}\right], \\
& \sigma=\frac{1}{D_{p e}}\left[\operatorname{cov}\left(u^{\prime}\left(c_{i}\right), e_{i}\right) \sum_{i=1}^{I} n_{i} \frac{\partial \tilde{x}_{i}}{\partial \theta}-\operatorname{cov}\left(u^{\prime}\left(c_{i}\right), x_{i}\right) \sum_{i=1}^{I} n_{i} \frac{\partial \tilde{x}_{i}}{\partial \sigma}\right. \\
& \left.-\sum_{i=1}^{I} n_{i}\left(\beta-\alpha_{i}\right) H_{\sigma i} \sum_{i=1}^{I} n_{i} \frac{\partial \tilde{x}_{i}}{\partial \theta}+\sum_{i=1}^{I} n_{i}\left(\beta-\alpha_{i}\right) H_{\theta i} \sum_{i=1}^{I} n_{i} \frac{\partial \tilde{x}_{i}}{\partial \sigma}\right],
\end{aligned}
$$

where

$$
D_{p e}=\mu\left[\sum_{i=1}^{I} n_{i} \frac{\partial \tilde{x}_{i}}{\partial \theta} \sum_{i=1}^{I} n_{i} \frac{\partial \tilde{e}_{i}}{\partial \sigma}-\sum_{i=1}^{I} n_{i} \frac{\partial \tilde{e}_{i}}{\partial \theta} \sum_{i=1}^{I} n_{i} \frac{\partial \tilde{x}_{i}}{\partial \sigma}\right] .
$$

If we assume that the cross derivatives are negligible, namely that $\partial \tilde{x} / \partial \sigma \rightarrow 0$ and $\partial \tilde{e} / \partial \theta \rightarrow 0$, we obtain:

$$
\begin{aligned}
\theta & =\frac{\sum_{i=1}^{I} n_{i}\left(\beta-\alpha_{i}\right) h_{x}\left(x_{i}, e_{i}\right)\left(\partial \tilde{x}_{i} / \partial \theta\right)-\operatorname{cov}\left(u^{\prime}\left(c_{i}\right), x_{i}\right)}{-\mu \sum_{i=1}^{I} n_{i}\left(\partial \tilde{x}_{i} / \partial \theta\right)}, \\
\sigma & =\frac{\sum_{i=1}^{I} n_{i}\left(\beta-\alpha_{i}\right) h_{e}\left(x_{i}, e_{i}\right)\left(\partial \tilde{e}_{i} / \partial \sigma\right)-\operatorname{cov}\left(u^{\prime}\left(c_{i}\right), e_{i}\right)}{-\mu \sum_{i=1}^{I} n_{i}\left(\partial \tilde{e}_{i} / \partial \sigma\right)} .
\end{aligned}
$$

11 That is $\left(\partial \tilde{x}_{i} / \partial \theta\right)<0$ and $\left(\partial \tilde{e}_{i} / \partial \sigma\right)<0$. 
The expressions for the optimal levels of the sin tax and the health subsidy include two terms: a Pigouvian term that corrects for the degree of myopia and an optimal tax term that measures the redistributive impact of the sin tax or the health subsidy. The first term of the numerator of (21) and (22) is the Pigouvian term found in (6) and (7) summed over all individuals with weights equal to the effect of the tax on individual demands of either $x_{i}$ or $e_{i}$. With both derivatives of demand functions negative, this term calls for a tax on sin good and a subsidy on health care.

The second term of the numerator of (21) and (22) reflects redistributive considerations. It depends on the concavity of $u$, the initial inequality of earnings and the correlation between $\alpha_{i}$ and $w_{i}$. With identical individuals, this term disappears and (21) and (22) reduce to (6) and (7). ${ }^{12}$ With different individuals and no correlation between $\alpha_{i}$ and $w_{i}$, the covariance will be negative in both equations, since richer people consume more of all goods $\left(x_{i}, c_{i}\right.$ and $\left.e_{i}\right)$ than poorer people with the same degree of myopia. This tends to increase the tax on the sin good and decrease the subsidy on health care, compared to the case with identical individuals. A positive correlation (between $\alpha_{i}$ and $w_{i}$ ) tends to reduce the consumption of $x_{i}$ of the high wage individuals. The sin good consumption now increases less fast with income and it may even decrease (when the myopia effect dominates the income effect). ${ }^{13}$ Consequently, the $\operatorname{cov}\left(u^{\prime}\left(c_{i}\right), x_{i}\right)$ will increase and may even become positive which leads to a smaller sin tax than with zero correlation. Intuitively, the redistributive benefits of sin taxes are mitigated or even reversed when 12 We have a first-best solution with $\mu=u^{\prime}\left(c_{i}\right)$.

13 There are many studies that look at the correlation between wealth and other behaviors or characteristics such as smoking or obesity. They show that there is a strong negative correlation between this characteristics and wealth (e.g. Stunkard and Sorensen, 1993). This literature provides admittedly indirect support for a positive correlation between $\alpha_{i}$ and $w_{i}$. The negative correlation between obesity or smoking and wealth can also be explained by factors other than myopia; for example for the case of obesity a negative correlation with wealth can also follow from differences in food prices according to fat content. 
the rich are less myopic. Either way, the absolute value of the covariance will tend to be larger the more concave is $u$ and the more unequal is the wage distribution. This will increase the sin tax when it is progressive (i.e., when $\left.\operatorname{cov}\left(u^{\prime}\left(c_{i}\right), x_{i}\right)<0\right)$ and decrease the tax when it is regressive (positive covariance).

The impact of a positive correlation on $\operatorname{cov}\left(u^{\prime}\left(c_{i}\right), e_{i}\right)$ is less clear: wealthier people buy less sin good, but they also better realize the importance of health care, so that the net impact on the amount of $e_{i}$ consumed is not easy to determine. These results are summarized in the following proposition.

Proposition 3 Consider the case of persistent errors where the policy tools are restricted to a linear sin tax, a linear subsidy on health and a lump sum transfer. If cross derivatives $\partial \tilde{x} / \partial \sigma$ and $\partial \tilde{e} / \partial \theta$ are negligible we have the following results:

a) The expressions for the optimal levels of $\theta$ and $\sigma$ are given by equations (21) and (22).

b) The level of the sin tax decreases as the correlation between $\alpha_{i}$ and $w_{i}$ increases (i.e., when wealthier individuals tend to be less myopic).

c) When $\operatorname{cov}\left(u^{\prime}\left(c_{i}\right), x_{i}\right)<0$ (resp. $\left.\operatorname{cov}\left(u^{\prime}\left(c_{i}\right), x_{i}\right)>0\right)$ the sin tax tends to be higher (lower) the more unequal the wage distribution and the more concave the utility function.

\section{Second-best with dual self}

Now we assume that the individuals realize after one period that they made a mistake and that the only corrective decision they can make is the choice of health expenditure. As in section II, we thus distinguish between the planned investment $e_{i}^{P}$ and the ex post choice $e_{i}$. The indirect utility function used by the social planner in its welfare maximization has to take into account these two values of $e_{i}$ which yield two values of $d_{i}$ (planned and effective). 
In the first period, the functions $x_{i}(\theta, \sigma, a), s_{i}(\theta, \sigma, a)$ and $e_{i}^{P}(\theta, \sigma, a)$ are obtained as the solution to

$$
\begin{aligned}
& -u^{\prime}\left(c_{i}\right)+u^{\prime}\left(d_{i}^{P}\right)=0, \\
& -u^{\prime}\left(c_{i}\right)(1+\theta)+\varphi^{\prime}\left(x_{i}\right)+\alpha_{i} h_{x}\left(x_{i}, e_{i}^{P}\right)=0, \\
& -u^{\prime}\left(d_{i}^{P}\right)(1+\sigma)+\alpha h_{e}\left(x_{i}, e_{i}^{P}\right)=0 .
\end{aligned}
$$

where $d_{i}^{P}=s_{i}-e_{i}^{P}(1+\sigma)>d_{i}=s_{i}-e_{i}(1+\sigma)$.

In the second period the effective demand for $e$ is defined by

$$
(1+\sigma) u^{\prime}\left(d_{i}\right)=\beta h_{e}\left(x_{i}, e_{i}\right)
$$

which yields $e_{i}$ as a function of $x_{i}$ and $s_{i}$. Substituting these variables by their expressions in terms of $(\theta, \sigma, a)$ yields

$$
e_{i}=f\left(s_{i}, x_{i}, e_{i}^{P}\right)=e_{i}(\theta, \sigma, a)
$$

The Lagrangian is given by

$$
\begin{aligned}
\mathcal{L}_{3}= & \sum_{i=1}^{I} n_{i}\left[u\left(w_{i}-s_{i}-x_{i}(1+\theta)+a\right)+\varphi\left(x_{i}\right)+u\left(s_{i}-(1+\sigma) e_{i}\right)\right. \\
& \left.+\beta h\left(x_{i}, e_{i}\right)-\mu\left(a-\theta x_{i}-\sigma e_{i}\right)\right],
\end{aligned}
$$

which is similar to $\mathcal{L}_{2}$ except that individual choices are now determined by (23), (24) and (26). The first-order conditions for the maximization of $\mathcal{L}_{3}$ are given in Appendix B. These can be rewritten as follows if we assume that the cross price effects are negligible:

$$
\begin{gathered}
\theta=\frac{1}{-\mu \sum_{i=1}^{I} n_{i}\left(\partial \tilde{x}_{i} / \partial \theta\right)}\left[-\operatorname{cov}\left[u^{\prime}\left(c_{i}\right), x_{i}\right]+\sum_{i=1}^{I} n_{i}\left[u^{\prime}\left(d_{i}\right)-u^{\prime}\left(d_{i}^{P}\right)\right] \frac{\partial \tilde{s}_{i}}{\partial \theta}\right. \\
\left.+\sum_{i=1}^{I} n_{i}\left[\beta h_{x}\left(x_{i}, e_{i}\right)-\alpha_{i} h_{x}\left(x_{i}, e_{i}^{P}\right)\right] \frac{\partial \tilde{x}_{i}}{\partial \theta}\right], \\
\sigma=\frac{1}{-\mu \sum_{i=1}^{I} n_{i}\left(\partial \tilde{e}_{i} / \partial \sigma\right)}\left[-\operatorname{cov}\left[u^{\prime}\left(c_{i}\right), e_{i}\right]+\sum_{i=1}^{I} n_{i}\left[u^{\prime}\left(d_{i}\right)-u^{\prime}\left(d_{i}^{P}\right)\right] \frac{\partial \tilde{s}_{i}}{\partial \sigma}\right] .
\end{gathered}
$$


The expression for the optimal level of the sin tax includes three terms: an optimal tax term that measures the redistributive impact of the sin tax, a "saving compensation" term (which accounts for the impact of the sin tax on the, otherwise insufficient, level of saving) and a Pigouvian term that corrects for the degree of myopia. The covariance term is the same as that in the single self scenario and reflects the equity concern of public policy. The second term of the numerator in (27) has the same sign as $\partial \tilde{s} / \partial \theta$. The intuition for this term goes as follows: individuals over-estimate their second period consumption $\left(d^{P}>d\right)$ since they under-estimate their health care needs. As a consequence, they do not save enough. To compensate for this, the tax will tend to be higher if it has a positive effect on saving. The third term of the numerator of (27) is the Pigouvian term found in equation (13) summed over all individuals with weights equal to the effect of the tax on individual demands of $x$. With $\alpha=0$ or $h_{x e}<0$, it is positive (as long as $\partial \tilde{x} / \partial \theta<0$ ). Equation (28) has a similar interpretation, except that only the first two terms are present (redistribution and savings compensation). There is no Pigouvian term here. ${ }^{14}$

In the case of identical individuals, (27) and (28) can be rewritten as:

$$
\begin{aligned}
\theta= & \frac{1}{\mu}\left[u^{\prime}\left(d^{P}\right)-u^{\prime}(d)\right] \frac{\partial \tilde{s} / \partial \theta}{\partial \tilde{x} / \partial \theta} \\
& +\frac{1}{\mu}\left\{(\alpha-\beta) h_{x}(x, e)+\alpha\left[h_{x}\left(x, e^{P}\right)-h_{x}(x, e)\right]\right\}, \\
\sigma= & \frac{1}{\mu}\left[u^{\prime}\left(d^{P}\right)-u^{\prime}(d)\right] \frac{\partial \tilde{s} / \partial \sigma}{\partial \tilde{e} / \partial \sigma} .
\end{aligned}
$$

Unlike in the case of persistent error, the first-best can no longer be achieved with the two considered instruments even when individuals are identical. The second part of the sin tax is the familiar Pigouvian term, expressed as in the first-best decentralization equation (14). The other term in both equations comes from our inability to control saving directly, which would be necessary to decentralize the first-best optimum. Saving 14 Recall that we are in the case where cross price effects are negligible so that $\sigma$ has no impact on $x$. 
can be indirectly controlled through the use of both $\theta$ and $\sigma$. If any of these instruments stimulates saving, this makes using it more desirable. These results are summarized in the following proposition.

Proposition 4 Consider the case of dual self where the policy tools are restricted to a linear sin tax, a linear subsidy on health and a lump sum transfer. If $\partial \tilde{x} / \partial \sigma \rightarrow 0$ and $\partial \tilde{e} / \partial \theta \rightarrow 0$, we have the following results:

a) The optimal tax formulas are given by equations (27) and (28).

b) Properties b) and c) of Proposition 3, remain valid in the dual self case.

c) The sin tax tends to be higher (and the health subsidy lower) the higher its impact on savings.

So far we have assumed that savings could not be taxed. Introducing $\tau$ as an extra instrument has a significant impact on the results only if all individuals are identical; in that case the linear instruments are sufficient to implement the first-best. However, with heterogeneous individuals this is not true and the qualitative results are not affected by the availability of a tax (or subsidy) on savings. ${ }^{15}$ In particular, the saving compensation term does not disappear from the expression because $\tau$ does not provide a perfect control of individual savings.

\section{Conclusion}

In this paper we have considered the case of sin goods that have delayed negative effects that individuals ignore at the time of consumption but acknowledge later. Individuals have then the possibility of partially compensating those negative effects by investing in

15 Except that with this extra instrument, the second-best solution will be close to the first-best outcome when the degree of heterogeneity is small. 
health care. Assuming a paternalistic government, we show that the first-best could be decentralized with a sin tax, a subsidy on saving and individualized lump sum transfers (or alternatively, by assuming identical individuals). In the second-best, individualized lump sum transfers are not available and the only available instruments are a linear sin tax and a linear subsidy on health care. We discuss the optimal second-best tax subsidy policy wherein distributive and corrective Pigouvian considerations are mixed.

We also consider the case of what we call persistent error, namely the case where individuals acknowledge the negative effects of their sinful consumption when it is too late to take any corrective action (i.e., after $e_{i}$ has been chosen). From an individuals perspective this latter case is formally equivalent to yet another setting which corresponds to what can be called persisting ignorance, where the individual never acknowledges the negative effects of his consumption.

The interaction between sin goods and health spending and its incidence on sin taxes is at the heart of this paper. We have seen that in the first-best the sin tax closely depends on the cross derivative $h_{x e}$. We have also shown that the impact of the possibility of making compensatory health expenditures on the size of the sin taxes depends on this same cross derivative. In the second best the degree of substitutability between $e$ and $x$ influences the sin tax in a more complex way. All the effects described in the first-best remain applicable (through the Pigouvian term which depends on $h_{x e}$ ). However, there are now many more avenues through which the interaction affects the optimal policy. Specifically, it affects the compensated derivatives as well as the covariance terms. Our results provide some general qualitative insight into these problems. To reach more specific and quantitative conclusions, empirical studies or at least calibrated simulations of our setting are necessary. 
In this paper we have focused on sin goods consumption. Our method could be used for other problems. For example, lack of physical exercises or hygiene in the first period of life which has delayed detrimental effects. These effects can be partially offset in the second period. Another example is overtime or moonlighting that lead to early disability. A fully rational individual would understand the importance of not abusing one's body when young to avoid regretful consequences later on in lifetime. The ingredients of these various situations are: behavior with delayed detrimental effects, myopia and possibility of partial compensation.

\section{Appendix}

\section{A First-order conditions for the maximization of $\mathcal{L}_{2}$}

Assuming interior solutions and making use of (16), (17) and (18), the FOCs of the social problem are given by:

$$
\begin{aligned}
\frac{\partial \mathcal{L}_{2}}{\partial a}= & \sum_{i=1}^{I} n_{i} u^{\prime}\left(c_{i}\right)+\sum_{i=1}^{I} n_{i}\left[h_{x}\left(x_{i}, e_{i}\right) \frac{\partial x_{i}}{\partial a}+h_{e}\left(x_{i}, e_{i}\right) \frac{\partial e_{i}}{\partial a}\right]\left(\beta-\alpha_{i}\right) \\
& -\mu \sum_{i=1}^{I} n_{i}\left[1-\theta \frac{\partial x_{i}}{\partial a}-\sigma \frac{\partial e_{i}}{\partial a}\right]=0 \\
\frac{\partial \mathcal{L}_{2}}{\partial \theta}= & -\sum_{i=1}^{I} n_{i} u^{\prime}\left(c_{i}\right) x_{i}+\sum_{i=1}^{I} n_{i}\left[h_{x}\left(x_{i}, e_{i}\right) \frac{\partial x_{i}}{\partial \theta}+h_{e}\left(x_{i}, e_{i}\right) \frac{\partial e_{i}}{\partial \theta}\right]\left(\beta-\alpha_{i}\right) \\
& +\mu \sum_{i=1}^{I} n_{i}\left[x_{i}+\theta \frac{\partial x_{i}}{\partial \theta}+\sigma \frac{\partial e_{i}}{\partial \theta}\right]=0 \\
\frac{\partial \mathcal{L}_{2}}{\partial \sigma}= & -\sum_{i=1}^{I} n_{i} u^{\prime}\left(d_{i}\right) e_{i}+\sum_{i=1}^{I} n_{i}\left[h_{x}\left(x_{i}, e_{i}\right) \frac{\partial x_{i}}{\partial \sigma}+h_{e}\left(x_{i}, e_{i}\right) \frac{\partial e_{i}}{\partial \sigma}\right]\left(\beta-\alpha_{i}\right) \\
& +\mu \sum_{i=1}^{I} n_{i}\left[e+\theta \frac{\partial x_{i}}{\partial \sigma}+\sigma \frac{\partial e_{i}}{\partial \sigma}\right]=0 .
\end{aligned}
$$




\section{B First-order conditions for the maximization of $\mathcal{L}_{3}$}

The FOCs are given by

$$
\begin{aligned}
\frac{\partial \mathcal{L}_{3}}{\partial a}= & \sum_{i=1}^{I} n_{i} u^{\prime}\left(c_{i}\right)+\sum_{i=1}^{I} n_{i}\left[u^{\prime}\left(d_{i}\right)-u^{\prime}\left(d_{i}^{P}\right)\right] \frac{\partial s_{i}}{\partial a} \\
& +\sum_{i=1}^{I} n_{i} \tilde{H}_{i} \frac{\partial x}{\partial a}-\mu \sum_{i=1}^{I} n_{i}\left[1-\theta \frac{\partial x_{i}}{\partial a}-\sigma \frac{\partial e_{i}}{\partial a}\right]=0 \\
\frac{\partial \mathcal{L}_{3}}{\partial \theta}= & -\sum_{i=1}^{I} n_{i} u^{\prime}\left(c_{i}\right) x_{i}+\sum_{i=1}^{I} n_{i}\left[u^{\prime}\left(d_{i}\right)-u^{\prime}\left(d_{i}^{P}\right)\right] \frac{\partial s_{i}}{\partial \theta} \\
& +\sum_{i=1}^{I} n_{i} \tilde{H}_{i} \frac{\partial x_{i}}{\partial \theta}+\mu \sum_{i=1}^{I} n_{i}\left[x_{i}+\theta \frac{\partial x_{i}}{\partial \theta}+\sigma \frac{\partial e_{i}}{\partial \theta}\right]=0 \\
\frac{\partial \mathcal{L}_{3}}{\partial \sigma}= & -\sum_{i=1}^{I} n_{i} u^{\prime}\left(d_{i}\right) e_{i}+\sum_{i=1}^{I} n_{i}\left[u^{\prime}\left(d_{i}\right)-u^{\prime}\left(d_{i}^{P}\right)\right] \frac{\partial s_{i}}{\partial \sigma} \\
& +\sum_{i=1}^{I} n_{i} \tilde{H}_{i} \frac{\partial x_{i}}{\partial \sigma}+\mu \sum_{i=1}^{I} n_{i}\left[e_{i}+\theta \frac{\partial x_{i}}{\partial \sigma}+\sigma \frac{\partial e_{i}}{\partial \sigma}\right]=0,
\end{aligned}
$$

where

$$
\tilde{H}_{i}=\beta h_{x}\left(x_{i}, e_{i}\right)-\alpha_{i} h_{x}\left(x_{i}, e_{i}^{P}\right) .
$$

As in section III we use $\partial \mathcal{L}_{3} / \partial a$ to obtain the compensated expressions of $\partial \mathcal{L}_{3} / \partial \theta$ and $\partial \mathcal{L}_{3} / \partial \sigma$

$$
\begin{aligned}
& \frac{\partial \tilde{\mathcal{L}}_{3}}{\partial \theta}=-\operatorname{cov}\left[u^{\prime}\left(c_{i}\right), x_{i}\right]+\sum_{i=1}^{I} n_{i}\left[u^{\prime}\left(d_{i}\right)-u^{\prime}\left(d_{i}^{P}\right)\right] \frac{\partial \tilde{s}_{i}}{\partial \theta} \\
&+ \sum_{i=1}^{I} n_{i} \tilde{H}_{i} \frac{\partial \tilde{x}_{i}}{\partial \theta}+\mu \sum_{i=1}^{I} n_{i}\left[\theta \frac{\partial \tilde{x}_{i}}{\partial \theta}+\sigma \frac{\partial \tilde{e}_{i}}{\partial \theta}\right]=0, \\
& \frac{\partial \tilde{\mathcal{L}}_{3}}{\partial \sigma}=-\operatorname{cov}\left[u^{\prime}\left(c_{i}\right), e_{i}\right]+\sum_{i=1}^{I} n_{i}\left[u^{\prime}\left(d_{i}\right)-u^{\prime}\left(d_{i}^{P}\right)\right] \frac{\partial \tilde{s}_{i}}{\partial \sigma} \\
&+\sum_{i=1}^{I} n_{i} \tilde{H}_{i} \frac{\partial \tilde{x}_{i}}{\partial \sigma}+\mu \sum_{i=1}^{I} n_{i}\left[\theta \frac{\partial \tilde{x}_{i}}{\partial \sigma}+\sigma \frac{\partial \tilde{e}_{i}}{\partial \sigma}\right]=0 .
\end{aligned}
$$

It is clear from the above that even with identical individuals, one cannot achieve the 
first-best with $\theta$ and $\sigma$ as instruments. Solving for $\theta$ and $\sigma$, we obtain

$$
\begin{aligned}
& \theta=\frac{1}{D_{d s}}\left\{\operatorname{cov}\left[u^{\prime}\left(c_{i}\right), x_{i}\right] \sum_{i=1}^{I} n_{i} \frac{\partial \tilde{e}_{i}}{\partial \sigma}-\operatorname{cov}\left[u^{\prime}\left(c_{i}\right), e_{i}\right] \sum_{i=1}^{I} n_{i} \frac{\partial \tilde{e}_{i}}{\partial \theta}\right. \\
& -\sum_{i=1}^{I} n_{i} \tilde{H}_{i} \frac{\partial \tilde{x}_{i}}{\partial \theta} \sum_{i=1}^{I} n_{i} \frac{\partial \tilde{e}_{i}}{\partial \sigma}+\sum_{i=1}^{I} n_{i} \tilde{H}_{i} \frac{\partial \tilde{x}_{i}}{\partial \sigma} \sum_{i=1}^{I} n_{i} \frac{\partial \tilde{e}_{i}}{\partial \theta} \\
& -\sum_{i=1}^{I} n_{i}\left[u^{\prime}\left(d_{i}\right)-u^{\prime}\left(d_{i}^{P}\right)\right] \frac{\partial \tilde{s}_{i}}{\partial \theta} \sum_{i=1}^{I} n_{i} \frac{\partial \tilde{e}_{i}}{\partial \sigma} \\
& \left.+\sum_{i=1}^{I} n_{i}\left[u^{\prime}\left(d_{i}\right)-u^{\prime}\left(d_{i}^{P}\right)\right] \frac{\partial \tilde{s}_{i}}{\partial \sigma} \sum_{i=1}^{I} n_{i} \frac{\partial \tilde{e}_{i}}{\partial \theta}\right\}, \\
& \sigma=\frac{1}{D_{d s}}\left\{\operatorname{cov}\left[u^{\prime}\left(c_{i}\right), e_{i}\right] \sum_{i=1}^{I} n_{i} \frac{\partial \tilde{x}_{i}}{\partial \theta}-\operatorname{cov}\left[u^{\prime}\left(c_{i}\right), x_{i}\right] \sum_{i=1}^{I} n_{i} \frac{\partial \tilde{x}_{i}}{\partial \sigma}\right. \\
& -\sum_{i=1}^{I} n_{i} \tilde{H}_{i} \frac{\partial \tilde{x}_{i}}{\partial \sigma} \sum_{i=1}^{I} n_{i} \frac{\partial \tilde{x}_{i}}{\partial \theta}+\sum_{i=1}^{I} n_{i} \tilde{H}_{i} \frac{\partial \tilde{x}_{i}}{\partial \theta} \sum_{i=1}^{I} n_{i} \frac{\partial \tilde{x}_{i}}{\partial \sigma} \\
& -\sum_{i=1}^{I} n_{i}\left[u^{\prime}\left(d_{i}\right)-u^{\prime}\left(d_{i}^{P}\right)\right] \frac{\partial \tilde{s}_{i}}{\partial \sigma} \sum_{i=1}^{I} n_{i} \frac{\partial \tilde{x}_{i}}{\partial \theta} \\
& \left.+\sum_{i=1}^{I} n_{i}\left[u^{\prime}\left(d_{i}\right)-u^{\prime}\left(d_{i}^{P}\right)\right] \frac{\partial \tilde{s}_{i}}{\partial \theta} \sum_{i=1}^{I} n_{i} \frac{\partial \tilde{x}_{i}}{\partial \sigma}\right\},
\end{aligned}
$$

where

$$
D_{d s}=\mu\left[\sum_{i=1}^{I} n_{i} \frac{\partial \tilde{x}_{i}}{\partial \theta} \sum_{i=1}^{I} n_{i} \frac{\partial \tilde{e}_{i}}{\partial \sigma}-\sum_{i=1}^{I} n_{i} \frac{\partial \tilde{e}_{i}}{\partial \theta} \sum_{i=1}^{I} n_{i} \frac{\partial \tilde{x}_{i}}{\partial \sigma}\right] .
$$




\section{References}

[1] Aronsson, T. and L. Thunstrom (2008), A note on optimal paternalism and health capital subsidies. Economics Letters 101, 241-242.

[2] Aronsson, T. and T. Sjogren (2009), Quasi-hyperbolic discounting and mixed taxation, Umea Economic Studies 778.

[3] Besley, T. (1988), A simple model for merit good arguments, Journal of Public Economics 35, 371-383.

[4] Blomquist, S. and L. Micheletto (2006), Optimal redistributive taxation when governments' and agents' preferences differ, Journal of Public Economics 90, 1215-1233.

[5] Camerer, C., S. Issacharoff, G. Loewenstein, T. O’Donoghue and M. Rabin (2003), Regulation for conservatives: behavioral economics and the case for "asymmetric paternalism", University of Pennsylvania Law Review 151, 1211-1254.

[6] Cremer, H., Ph. De Donder, D. Maldonado and P. Pestieau (2009), Forced saving, redistribution and non linear social security scheme, Southern Economic Journal 76, $86-98$.

[7] Cremer, H., Ph. De Donder, D. Maldonado and P. Pestieau (2007), Voting over the size and type of social security when some individuals are myopic, Journal of Public Economics 91, 2041-2061, 2007.

[8] Cremer H., Ph. De Donder, D. Maldonado and P. Pestieau (2008), Habit formation and labor supply, CORE DP 2008/38. 
[9] Fong, G. T., D. Hammond, F. Larou, M. Cummings, R. Boland and H. Ross (2004), The non universal experience of regret among smokers in four countries, Nicotine and Tobacco Research 6, S341-S351.

[10] Gruber, J. and B. Koszegi (2001), Is addiction 'rational'? theory and evidence, Quarterly Journal of Economics 116, 1261-1303.

[11] Gruber J. and B. Koszegi (2004), Tax incidence when individuals are timeinconsistent: the case of cigarette excise taxes, Journal of Public Economics 88, 1959-1987.

[12] Harris, J. (1999), Communicable diseases, lifestyles and personal responsibility, Ethics and rights, unpublished.

[13] Haavio, M. and K. Kotakorpi (2009), The political economy of sin taxes. CESifo Working paper 2650.

[14] Laibson, D. (1997), Hyperbolic discounting and golden eggs, Quarterly Journal of Economics 112, 443-477.

[15] Musgrave, R. (1959), The Theory of Public Finance, McGraw-Hill, New York.

[16] O'Donoghue, T. and M. Rabin (1999), Procrastination in preparing for retirement, in H. J. Aaron (ed.), Behavioral Dimensions of Retirement Economics, Brookings Institution, Washington, D.C.

[17] O'Donoghue, T. and M. Rabin (2003), Studying optimal paternalism, illustrated by a model of sin taxes, American Economic Review (Papers and Proceedings) 93, 186-191. 
[18] O'Donoghue, T. and M. Rabin (2006), Optimal sin taxes, Journal of Public Economics $90,1825-1849$.

[19] Stunkard, A. J. and T. Sorensen (1993), Obesity and socioeconomic status, The New England Journal of Medicine 329, 1036-1037.

[20] Thaler, R. and C. Sunstein (2003), Behavioral economics, public policy, and paternalism, American Economic Review (Papers and Proceedings) 93, 175-179. 